\title{
DENSITY IRREGULARITIES IN THE PLASMASPHERE BOUNDARY LAYER: CLUSTER OBSERVATIONS IN THE DUSK SECTOR
}

\author{
P.M.E. Décréau ${ }^{1}$, E. Le Guirriec ${ }^{1}$, J.L. Rauch ${ }^{1}$, J. G. Trotignon ${ }^{1}$, P. Canu ${ }^{2}$, F. Darrouzet ${ }^{3}$, J. Lemaire ${ }^{3}$, A. Masson ${ }^{4}$, \\ F. Sedgemore ${ }^{5}$ M. André \\ ${ }^{1} L P C E$, CNRS-Université d'Orléans, $3 A$ Avenue de la Recherche Scientifique, 45071 Orléans Cédex02, France \\ ${ }^{2}$ CETP, CNRS-Université VSQ, 10-12 Avenue de l'Europe, 92130 Vélizy, France \\ ${ }^{3}$ Belgian Institute for Space Aeronomy, IASB-BIRA, 3 Avenue. Circulaire, B-1180 Brussels, Belgium \\ ${ }^{4} R S S D$, ESA, Keplerlaan 1, 2200 AG,Noordwijk,The Netherlands \\ ${ }^{5}$ DSRI, Juliane Maries Vej. 30, DK-2100, Copenhaguen Oe, Danemark \\ ${ }^{6}$ IFRI, Uppsala division, Box 537, SE-751 21 Uppsala, Sweden
}

\begin{abstract}
We present observations of plasma density structures crossed by the CLUSTER spacecraft constellation near orbit perigee, with the spacecraft located in the vicinity of the plasmapause boundary. Although the constellation is not arranged as a perfect tetrahedron, the four point measurements reveal interesting properties of density structures, unknown before the 4-points in-situ observations from the CLUSTER mission. The set of plasma density profiles shown are derived from the EFW and WHISPER instruments observations in the dusk sector of the plasmasphere region. They yield new insights into: (i) comparative along and cross-field dimensions, and (ii) the dynamics of small-scale structures. They illustrate the great opportunity offered by the CLUSTER mission to resolve new challenges in magnetospheric physics, including a clearer understanding of the physics involved in the dynamics of the plasmasphere and the formation of its outer boundary: the plasmapause.
\end{abstract}

\section{INTRODUCTION}

One of the main objectives of the multi-spacecraft CLUSTER mission is the study of small-scale plasma structures. The grounds for using four measurement points is to overcome the limitations of a single spacecraft, or even of a pair, in resolving the spatio-temporal evolution of plasma structures; indeed such information is crucial for understanding the underlying physical mechanisms, involved in the formation of new plasmapause density gradients. We present here case studies of density irregularities observed by CLUSTER in the plasmapause region. We show how a comparison of the four measured density profiles can improve our in-situ perception and knowledge of this key magnetospheric region.

Recognizing that a four spacecraft formation is not able to fully resolve the evolution of a plasma structure in the general case, we follow here two main approaches. The first one considers the simple problem of a rigid planar structure drifting at constant velocity during the time interval when it is crossed successively by the four spacecraft. Under such circumstances, the speed $\mathrm{V}$ along boundary normal, and corresponding direction $\mathbf{n}$, can be determined from the so called timing analysis method. The method relies on the equation $\mathbf{r}_{\mathrm{n}} \cdot \mathbf{n}=\mathrm{V} \mathrm{t}_{\mathrm{n}}$, where $\mathbf{r}_{\mathrm{n}}$ and $t_{\mathrm{n}}$ are the differential positions and times of crossing by three spacecraft relative to position and time of crossing by the fourth spacecraft chosen as reference. This method has proven to be successful in a number of studies carried on by CLUSTER (see for instance Dunlop et al., 2002). The second approach compares simply the shape of the four density profiles and identifies similar features in those profiles as signature of a structure coherent in time and/or space. This is facilitated by plotting density variations as a function of the L parameter. Relative spatio-temporal positions of identified features can further be used to derive useful characteristic dimensions.

The paper is divided in two main parts. The first part presents the general context: density measurements, orbital properties, general features observed in the Plasmasphere Boundary Layer (PBL, Carpenter, 2004). The second part presents detailed observations of two density structures, and some discussions about the physical processes implied. 


\section{GENERAL CONTEXT}

\section{Measurement techniques}

The density observations discussed in this paper are obtained by combining two instruments of CLUSTER: EFW (Gustafsson et al., 1997) and WHISPER (Décréau et al., 1997). Spacecraft potential values Vsc derived at a high time resolution by EFW follow the electron density $\mathrm{N}_{\mathrm{e}}$ variations in a given plasma regime (Laakso and Pedersen, 1998). They can be calibrated by WHISPER measurements (Moullard et al., 2002). Plasma frequency variations discussed in this paper are obtained from measured values of Vsc and the empirical parametric relationship: $\mathrm{Ne}_{\mathrm{e}}=\mathrm{A} * \mathrm{Vsc}_{\mathrm{SC}}{ }^{-\mathrm{B}}$. The constant $\mathrm{A}$ and $\mathrm{B}$ are obtained by fitting data subsets of Vsc measurements and of $\mathrm{N}_{\mathrm{e}}$ values derived by the WHISPER sounder at a lower time resolution (Trotignon et al., 2001).

\section{Orbitography}

CLUSTER travels on a polar orbit of large eccentricity (4 and 19.5 Earth radii respectively at perigee and apogee). During main manoeuvres (activated at least once a year), the spacecraft orbits are adjusted in order for the constellation to form a perfect tetrahedron of prescribed dimension usually in the cusp or close to the magnetopause.
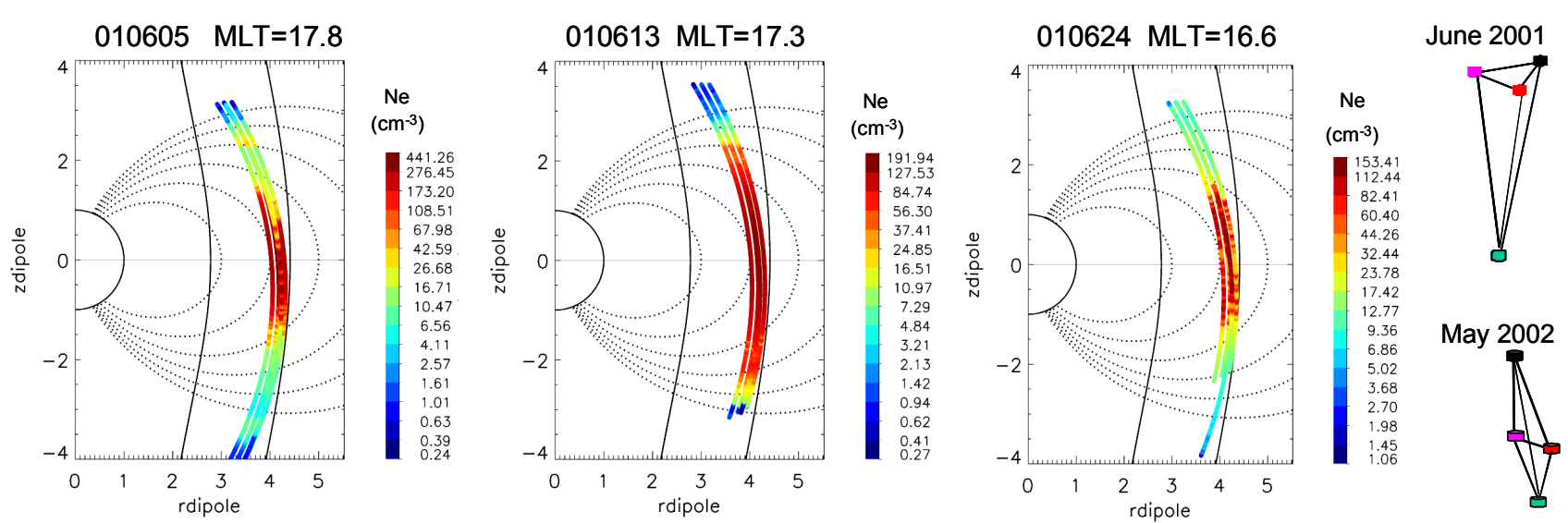

Fig.1. Colour coded density values measured at three perigee passes in the dusk sector, plotted along the spacecraft orbits in the SM coordinate system. C4 travels at the lowest altitudes, C1 at the highest ones. At intermediate altitudes, the track of C3 hides that of C2, taken about 45 minutes earlier. Dotted curves are dipole field lines (from $L=3$ to $L=8$ ). Solid curves are cuts of Roche limit surfaces. Constellation configurations close to perigee are sketched on the right (C1 in black, C2 in red, C3 in green and C4 in purple), respectively for the June 2001 epoch and for the May 2002 epoch (event of figure 5).

This results in an elongated constellation shape at perigee, where the plasmasphere region is encountered. In figure 1 each panel displays, for a given perigee pass, colour coded density values measured at successive spacecraft positions along the four orbit tracks. The orbit tracks are plotted in a meridian plane of the solar magnetic (SM) coordinate system (Russell, 1971), where the abscissa $r$ measures the distance of the spacecraft to the dipole axis and the ordinate $\mathrm{z}$ measures the distance of the spacecraft to the magnetic dipole equator. Such a presentation erases the local time differences at the various spacecraft positions, and only three orbit tracks appear as being distinct. The shape of Cluster configuration is sketched on the right of the figure, respectively top right for large average separation (June 2001) and bottom right for small separation (May 2002). In both cases, the largest separation $(\sim 10000 \mathrm{~km}$ in June $2001, \sim 300 \mathrm{~km}$ in May 2002) is aligned roughly along the orbit, tangent to the magnetic field direction at the equator. Across the orbit, the average separation is $\sim 2000 \mathrm{~km}$ in June 2001, $60 \mathrm{~km}$ in May 2002. The shape is less regular at large separation, three spacecraft, $\mathrm{C} 1, \mathrm{C} 2$ and $\mathrm{C} 4$, being grouped as a trio, than at small separation.

Dipole field lines are plotted from $\mathrm{L}=3$ to 8 (dotted lines), where the McIlwain parameter L (McIlwain, 1961) identifies a surface of cylindrical symmetry with respect to the dipole axis. Real field line orientations measured onboard by the FGM instrument (Balogh et al., 1997) deviate from this representation, which nevertheless guides the eye to recognize global features. It takes about 1 hour 40 minutes for a spacecraft to travel from South to North between the two orbit positions located at $\mathrm{L}=6$. MLT values at perigee are listed at the top of each orbit plot. They are decreasing from South to North, of about 1.8 hours between the two orbit positions located at $\mathrm{L}=6$.

\section{General topology}

Several interesting features appear clearly on figure 1, illustrating a general behaviour: 
1) the major density structures are globally field aligned. This is particularly true for the rather sharp density gradients typical of the plasmapause knee ('yellow' density levels in the $13^{\text {th }}$ June 2001 plots). It is also the case for density features separated from the main plasmasphere body as viewed on an orbit cut, which does not mean that the feature is necessarily detached (see example at $\mathrm{L}=6$, in the $5^{\text {th }}$ June 2001 plot, northern hemisphere),

2) a large number of density irregularities (10 to $200 \mathrm{~cm}^{-3} \mathrm{~N}_{\mathrm{e}}$ levels, typical density variations observed at plasmapause) are observed on some orbits $\left(5^{\text {th }}\right.$ and $24^{\text {th }}$ June 2001). The region encompassing those irregularities appears as rather large (entire orbit segment below $\mathrm{L} \sim 5$ ), roughly centred on the magnetic equator. This region has recently been called the Plasmasphere Boundary Layer (Carpenter, 2004).

The solid lines highlight two positions of the cylindrically symmetric Roche limit surface, or Zero Parallel Force (ZPF) surface if the angular velocity of the plasma would be 3 times larger than the corotation velocity (inner curve) and 1.5 times the corotation velocity (outer curve). According to the theory outlined by Lemaire (1999, 2001) the part of the plasmasphere outside this limit is convectively unstable, leading to the formation of quasiinterchange modes (see Ferrière et al., 2001) with a non-zero component of the k-vector parallel to the magnetic field lines. This produces small-scale density irregularities and leads to plasmapause formation via erosion in the post-midnight MLT sector, where the plasma angular velocity is the largest and the ZPF surface closest to Earth. André (2004) has calculated that the characteristic time for this type of instability is of the order of 10 minutes.

It happens that the Cluster orbit below $\mathrm{L} \sim 5$ is roughly aligned with a Roche limit surface, such that a large orbit segment will encounter similar unstable conditions if the surface happens to meet the orbit position, i.e. at plasma angular velocities about twice that of co-rotation. The presence of irregularities along large orbit segments is compatible with the hydrodynamic instability (HI) theory proposed by Lemaire. Let's hope that future studies will enable us: (i) to follow the formation of irregularities in the post-midnight sector and during magnetic substorms, where and when they are supposed to form; (ii) to identify how these density irregularities are convected away from the unperturbed co-rotating inner part of the plasmasphere, to be found later in the dusk sector.

\section{DETAILED OBSERVATIONS}

\section{Observations of the plasmapause region when spacecraft are widely separated}

Large separations are linked to large time intervals between spacecraft when crossing the same region of space. In June 2001, three spacecraft (C1, C2, C4) were within $2000 \mathrm{~km}$ of each other (sketch of Figure 1); C3 was significantly farther (at a distance of about $10000 \mathrm{~km}$ from the trio). The perigee is then at 17.3 MLT.

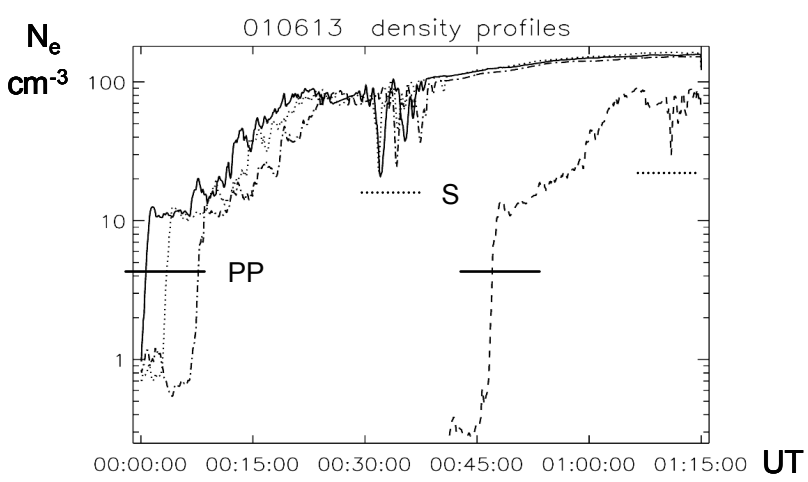

Fig.2. Compared density time profiles obtained on spacecraft C1 (solid line), C2 (dotted line), C3 (dashed line) and C4 (dash-dotted line), at the inbound leg of the 13 June 2001 pass. The analysed features, $P P$ and $S$, are pointed.

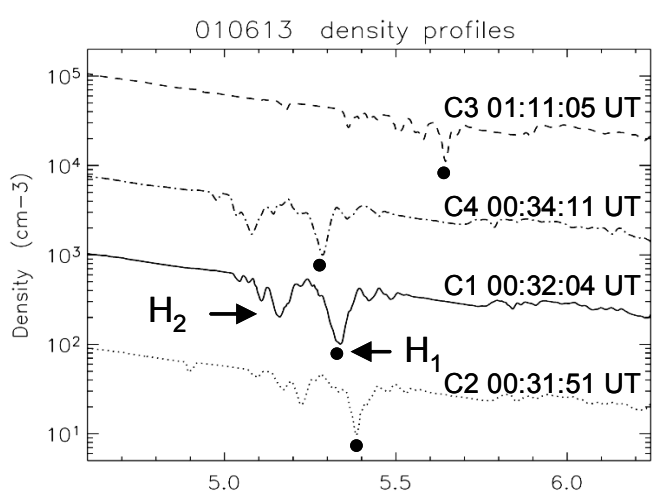

$\mathrm{R}_{\mathrm{eq}}$

Fig.3. Details of the structure $S$ : density versus $R_{\text {eq }}$ parameter. Density values have been multiplied by 10 for $C 1$, 100 for $C 4$ and 1000 for C3. Time runs from right to left. UT values are given at pointed $H_{1}$ dips.

Such an elongated configuration can be used for testing the stability of large scale boundaries. Using the timing analysis method described in the introduction, we have analyzed the motion of the plasmapause (PP) crossed around 00:05 UT by the C124 trio and 40 minutes later by C3 (as shown in Figure 2). This requires three conditions to be met: planarity, rigidity and velocity constancy. The four crossing points form a tetrahedron of reasonable characteristics, leading to a satisfying precision $(\sim 5 \%)$ both on orientation and magnitude of drift velocity. This tetrahedron is inscribed within a sphere of $\sim 1000 \mathrm{~km}$ radius, such that the requirement of planarity of the travelling boundary needs to be satisfied only at that scale. The position of the crossings are shown in figure 1 (middle panel) by the yellow region at $\mathrm{L} \sim 8$, southern hemisphere. There, the magnetic shell curvature radius is large enough (about $5 \mathrm{R}_{\mathrm{E}}$ ) for planarity to be satisfied locally. Concerning the rigidity, we note a striking similarity between the four density profiles displayed in Figure 2 (discarding small scale features). The thickness of the overall boundary 
layer and density distribution within, which are not varying significantly from one pass to the other, can qualify both as 'rigid' and travelling at a roughly constant velocity. The timing analysis indicates that the plasmapause velocity is low: $0.33 \mathrm{~km} \mathrm{~s}^{-1}$ in the GSE system (the total displacement over the large time interval studied is only $910 \mathrm{~km}, \sim 0.15 \mathrm{R}_{\mathrm{E}}$ ). The respective components of the boundary unit normal are $0.78,0.26$ and 0.56 . This vector is not oriented parallel to the local meridian plane (the $\mathrm{X}$ component is not small), as could be expected from an axisymmetric plasmapause. Considering that the boundary is encountered at a large $L$ value $(L=8)$ in the dusk sector, the likely explanation is that the plasmasphere expands in a bulge along the $\mathrm{Y}$ axis. The bulge is crossed by Cluster on its night-side flank, aligned with a magnetic shell oriented roughly along the 18 MLT meridian plane. We have tested the likeness of our interpretation and assumptions against independent information: the DC magnetic field vectors measured by the FGM instrument at the four crossing points. The four vectors have the same orientation within $\pm 2^{\circ}$ (confirming planarity). They are all perpendicular to the estimated boundary normal (within $\pm 2^{\circ}$ ), confirming the view of a plasmapause locally aligned with a magnetic shell.

After 00:30 UT, small scale structures (S) with large density indentations show up in the electron density versus time profiles of Figure 2 (density scales are shifted). In order to facilitate the comparison of the four profiles, we can plot the $\mathrm{N}_{\mathrm{e}}$ values measured at various positions as a function of the L parameter. Such an approach is directly relevant to in-situ observations when they are obtained by quasi equatorial missions, like ISEE 1 . In the case of Cluster, we make use of a magnetic field model, combining IGRF95 and Tsyganenko-96 (Tsyganenko and Stern, 1996). We follow the magnetic field line provided by the model from the spacecraft position to the equator (identified by a minimal magnetic field strength value), using the Unilib library (see reference list). The geocentric distance of this equatorial point, which we call $\mathrm{R}_{\mathrm{eq}}$ and which we express in Earth radii, plays the same role as the $\mathrm{L}$ value. As this paper deals mainly with small structures, we ignore the density variation with altitude in a magnetic tube of constant flux. All density values presented are raw in-situ measurements.

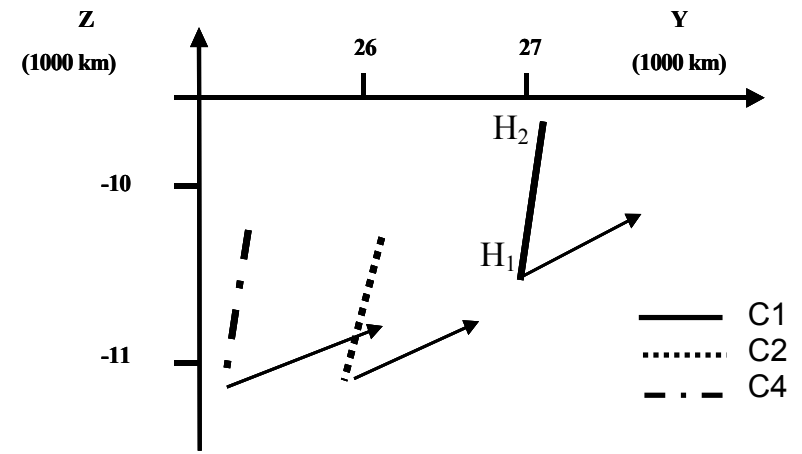

Fig. 4. Case event of 13 June 2001. Position of the density structure $\mathrm{H}_{1} \mathrm{H}_{2}$ (line style as in Figure 2) in a meridian plane of the SM coordinate system. The arrows indicate the respective magnitudes (350 $n T$ for $C 1)$ and orientation of the measured magnetic field vectors.
The details of the indentation crossed around 00:30 UT by the $\mathrm{C} 1, \mathrm{C} 2, \mathrm{C} 4$ trio, are displayed in Figure 3. They point out the same density signature, referred to below as $\mathrm{H}_{1} \mathrm{H}_{2}$ : a pair of density holes separated by roughly $0.2 \mathrm{R}_{\mathrm{E}}$ along the $\mathrm{R}_{\mathrm{eq}}$ parameter. In Figure 3, time runs from right to left and from bottom to up: $\mathrm{C} 2$ crosses the structure the first, $\mathrm{C} 113 \mathrm{~s}$ later $\left(\mathrm{H}_{1}\right.$, the first encountered density hole, is chosen as marker), C4 $127 \mathrm{~s}$ after that, and finally $\mathrm{C} 3$, roughly 40 minute after the trio. The orbit elements crossed by $\mathrm{C} 1, \mathrm{C} 2$ and $\mathrm{C} 4$ between the density holes $\mathrm{H}_{1} \mathrm{H}_{2}$ are located in almost the same meridian plane of the SM coordinate system (differences in longitude are less than one degree), which happens to be close to the YZ plane. In figure 4, those elements are plotted as line segments of the YZ plane. The three DC magnetic field vectors measured by the FGM instrument, plotted at the $\mathrm{H}_{1}$ markers, are quasi parallel to this plane (at an angle below $3^{\circ}$ ). In this particular configuration, figure 4 shows that the three spacecraft, which are less than $350 \mathrm{~km}$ apart in the third dimension (along the $\mathrm{X}$ axis) are roughly aligned along a direction roughly parallel to the magnetic field direction. They recognize the same cross-field structure, within a short time interval.

We can now discuss the dimension of the double indentation structure. In the plane of figure 4 (YZ plane of the SM coordinate system), its overall size along the field direction is $2000 \mathrm{~km}$ or more, whereas the distance between the field lines respectively along $\mathrm{H}_{1}$ holes and $\mathrm{H}_{2}$ holes is about $800 \mathrm{~km}$. The field line distance of smaller irregularities is below $100 \mathrm{~km}$. In the third dimension - in longitude - we can only state that the structure is larger than the maximal spacecraft separation, i.e. about $350 \mathrm{~km}$.

Another interesting piece of information derived from the multipoint measurements is the time life of the well defined density holes observed. A detailed inter-comparison of the three similar density profiles show actually slight differences between them, indicating either that there are differences over even smaller dimensions, or that the structure has evolved in time, over less than one minute time interval. Note that the profile measured by $\mathrm{C} 3$ is different, displaying shallower indentations (see figure 3). This could be a temporal effect, due to refilling (both magnetic feet are illuminated at ionospheric altitude), or to cross-field interchange motion. Alternatively, it could be a longitude effect (C2, C4, C1 and $\mathrm{C} 3$ are placed at increasing respective longitudes, within one degree total).

\section{Observations of the plasmapause region when the spacecraft are close to each other}

For a duration of about 4 months in 2002, the CLUSTER configuration has been adjusted to small separations, while the perigee was located in the midnight to dusk local time sector. Preliminary analysis confirms here also the 
presence of frequent small scale density irregularities in the vicinity of the plasmapause and inside the plasmasphere (Darrouzet et al., 2004); some of them can clearly be identified as the same entities on all four density profiles. We can in such cases apply the timing analysis, as done above.

Figure 5 displays detailed electron density profiles as a function of $R_{\text {eq }}$ at the plasmapause discontinuity observed the $9^{\text {th }}$ of May 2002 on the inbound leg near L $=6$, around 19 MLT. There, the orbit track and the magnetic field lines make an angle of about $60^{\circ}$, hence successive field lines at decreasing $\mathrm{R}_{\mathrm{eq}}$ are crossed rapidly: $1 \mathrm{R}_{\mathrm{E}}$ range in about 10 minutes. The four profiles show density values fluctuating between two smooth levels reached respectively in the high and low $\mathrm{R}_{\text {eq }}$ range, a typical situation in the outer plasmasphere region (as illustrated in Lemaire and Gringauz, 1998, chapter 2).

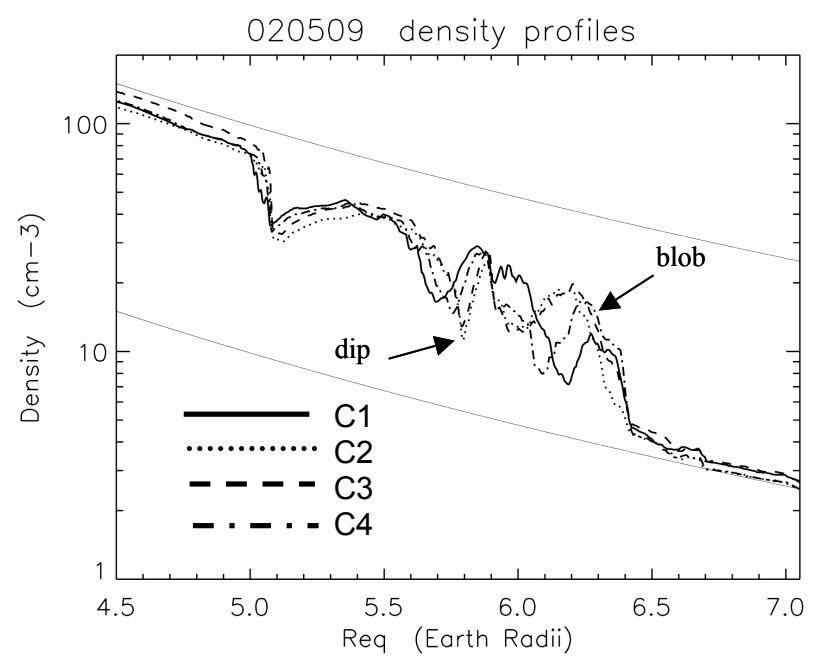

Fig. 5. Details of the electron density profiles versus $R_{e q}$ parameter measured on $9^{\text {th }}$ May 2002. Line code as in figure 2. The two thin lines are $R_{e q}{ }^{-4}$ profiles fitting observations respectively at low and high $R_{\text {eq }}$ values. Arrows point to structures discussed in text.

We have analysed the motion of the density dip observed at $R_{\text {eq }}=5.7$ to 5.8 via timing analysis. In this case of a small constellation (largest separation of $350 \mathrm{~km}$ at traversal points in the moving plane), the assumption of a planar boundary seems justified. The exercise yields velocity components of respectively $-2,0.7,0.5 \mathrm{~km} \mathrm{~s}^{-1}$ in the GSE co-ordinate system, indicating that co-rotation $\left(\sim 2 \mathrm{~km} \mathrm{~s}^{-1}\right.$ at this position) is the major mechanism involved in the plasma motion. On the other hand, the local orientation of magnetic field is measured at an angle of $\sim 30^{\circ}$ with the boundary. If the conditions for applying timing analysis are met, part of the motion is parallel to field line. This opens the possibility of hydrodynamic instability (HI) with a parallel component of centrifugal force. The plasma interchange velocity value according to the HI model can be evaluated from the formula listed in Lemaire and Gringauz, 1998, p. 263. For the density irregularities shown in Figure 5 above Req $=5.5$ (dip and blob with $\Delta \mathrm{n} \sim$ $50 \mathrm{~cm}^{-3}$ ) it amounts to a value of about $0.2 \mathrm{~km} \mathrm{~s}^{-1}$. If interchange is indeed at work, the corresponding motion of irregularities is too slow to be distinguished from co-rotation in this case, and we cannot conclude.

\section{SUMMARY AND DISCUSSION}

The configuration of CLUSTER orbits allows the four platforms to cross the outer region of the plasmapause, and has revealed large and small-scale patchiness of the plasma distribution in this transition region. The small separation (from $100 \mathrm{~km}$ up to $12000 \mathrm{~km}$ ) between the four spacecraft, combined with the very high time resolution (less than $3 \mathrm{~s}$ ) of the WHISPER and EFW observations, offer unprecedented opportunities to study in greatest details the small-scale irregularities in the narrow region of the plasmapause boundary layer.

In this study, it has been shown that the plasma irregularities observed at the plasmasphere boundary have dimensions along magnetic field larger than $2000 \mathrm{~km}$ (at least in the case event analysed), cross-field dimensions ranging from less than $20 \mathrm{~km}$ to more than $350 \mathrm{~km}$, and a wide range of lifetimes (from 10 seconds to several tens of minutes). Drift velocities have been derived from timing analysis, demonstrating - if simple assumptions are met - the slow motion of both a small and a large-scale density discontinuity. The results presented above constitute a first step toward further studies of plasma irregularities at the plasmapause boundary layer. In particular toward testing if plasmapause density gradients are forming due to plasma interchange instability (Lemaire, 1999, 2001). 


\section{ACKNOWLEDGMENTS}

The authors gratefully acknowledge the ESA teams at ESOC, Germany, and JSOC, UK, and the WEC operation team (University of Sheffield, UK), for their efficient work in operating the four spacecraft and four sets of WEC instruments. The Whisper team thanks CNRS and CNES, France, for their continuous support of the experiment. J. Lemaire and F. Darrouzet acknowledge the support by the Belgian Federal Science Policy Office through the ESA/PRODEX-7 CLUSTER project.

\section{REFERENCES}

André, N., (personal communication), 2004.

Balogh, A., M. W. Dunlop, S. W. H. Cowley, D. J. Southwood, J. G. Thomlinson, and the Cluster magnetometer team, The Cluster magnetic field investigation, Space Science Reviews, 79, 65, 1997.

Carpenter, D. L., Remote sensing the Earth's Plasmasphere, Radio Science Bulletin, No. 304, 13, 2004.

Darrouzet, F., Décréau, P., De Keyzer, J., Masson, A., Gallagher, D., Santolik, O., Sandel, B., Trotignon, J.-G., Rauch, J.-L., Le Guirriec, E., Canu, P., Sedgemore, F., André, M., and J. Lemaire, Density structures inside the plasmashere,: Cluster observations, Ann. Geophys., 22, 2577-2585, 2004.

Décréau, P. M. E., P. Fergeau, V. Krasnosels'kikh, M. Lévêque, Ph. Martin, O. Randriamboarison, F. X. Sené, J.G. Trotignon, P. Canu, P. B. Mögensen, and Whisper investigators, Whisper, a resonance sounder and wave analyser: performances and perspectives for the Cluster mission, Space Science Reviews, 79, 157-193, 1997.

Dunlop, M. W., A. Balogh, K.-H. Glassmeier, Four-point Cluster application of magnetic field analysis tools: The discontinuity analyzer, J. Geophys. Res., 107, No. A11, 1385, doi:10.1029/2001JA005089, 2002.

Ferrière, K. M., Zimmer, C. and M. Blanc, Quasi-interchange modes and interchange instability in rotating magnetospheres, J. Geophys. Res., 106, 327, 2001.

Gutafsson, G., R. Boström, B. Holback, G. Holmgren, A. Lundgren, K. Stasiewicz, L. Ahlen, F. S. Mozer, D. Pankow, P. Harvey, P. Berg, R. Ulrich, A. Pedersen, R. Schmidt, A. Butler, A. W. C. Fransen, D. Klinge, M. Thomsen, C.G. Fälthammar, P.-A. Lindqvist, S. Christenson, J. Holtet, B. Lybekk, T.A. Sten, P. Tanskanen, K. Lappalainen and J. Wygant, The electric field and wave experiment for the Cluster mission, Space Science Reviews, 79, 137-156, 1997.

Laakso, H., and A. Perdersen, Ambient electron density derived from differential potential measurements, in Measurements Techniques in Space Plasmas, Edited by P. Pfaff, J. Borovsky, and D. Young, AGU Monograph 102, pp. 49-54, AGU Washington DC, 1998.

Lemaire, J., Hydrostatic equilibrium and convective stability in the plasmasphere, Journal of Atmospheric and Solar-Terrestrial Physics, 61, 867-878, 1999.

Lemaire, J., The formation of the light-ion-trough and peeling off the plasmasphere, Journal of Atmospheric and Solar-Terrestrial Physics, 63, 1285-1291, 2001.

McIlwain, C. E., Coordinates for mapping the distribution of magnetically trapped particles, J. Geophys. Res., 66 , 3681-3691, 1961.

Moullard, O., Masson, A., Laakso, H., Parrot, M., Décréau, P., Santolik, O., and André, M., Density modulated whistler mode emissions observed near the plasmapause, Geophys. Res Lett., 29(19), 10.1029/2002 GL015101, 2002.

Lemaire, J.F., and K. I. Gringauz, The Earth's plasmasphere, with contributions from D. L. Carpenter and V. Bassolo, Cambridge University Press, Cambridge (ISBN 0.521.43091.7), 1998.

Russell, C. T., Geophysical Coordinate Transformations, Cosmic Electrodynamics, 2, 184-196, 1971.

Tsyganenko, N. A., and D. P. Stern, Modeling the global magnetic field of the large-scale Birkeland current systems, J. Geophys. Res., 101(A12), 27187-27198 (96JA02735), 1996.

Trotignon, J.-G., P. M. E. Décréau, J.-L. Rauch, O. Randriamboarison, V. Krasnoselskikh, P. Canu, H. Alleyne, K. Yearby, E. Le Guirriec, H. C. Séran, F. X. Sené, Ph. Martin, M. Lévêque, P. Fergeau, How to determine the thermal electron density and the magnetic field strength from ,the CLUSTER/WHISPER observations around the Earth, Ann. Geophys., 19, 1711-1720, 2001.

UNILIB Library of routines for magnetospheric applications, http://www.oma.be/NEEDLE/unilib.php/20x/index.html.

E-mail address of P.M.E. Décréau: pdecreau@cnrs-orleans.fr

Manuscript received December 2002 ; revised March 2005 ; accepted August 2005. 